Check for updates

Cite this: Phys. Chem. Chem. Phys., 2018, 20, 16167

Received 5th March 2018 Accepted 25th April 2018

DOI: $10.1039 / c 8 c p 01442 f$

rsc.li/pccp

\section{The electronic structure and the nature of the chemical bond in $\mathrm{CeO}_{2}$}

\author{
Konstantin I. Maslakov, (iD a Yury A. Teterin, ${ }^{\text {ab }}$ Mikhail V. Ryzhkov, (D) c \\ Aleksej J. Popel, iD *d Anton Yu. Teterin, ${ }^{b}$ Kirill E. Ivanov, ${ }^{b}$ Stepan N. Kalmykov, ${ }^{a b}$ \\ Vladimir G. Petrov, ${ }^{a}$ Peter K. Petrov ${ }^{e}$ and Ian Farnan ${ }^{d}$
}

\begin{abstract}
The X-ray photoelectron spectral structure of $\mathrm{CeO}_{2}$ valence electrons in the binding energy range of 0 to $\sim 50 \mathrm{eV}$ was analyzed. The core-electron spectral structure parameters and the results of relativistic discrete-variational calculations of $\mathrm{CeO}_{8}$ and $\mathrm{Ce}_{63} \mathrm{O}_{216}$ clusters were taken into account. Comparison of the valence and the core-electron spectral structures showed that the formation of the inner (IVMO) and the outer (OVMO) valence molecular orbitals contributes to the spectral structure more than the many-body processes. The $\mathrm{Ce} 4 \mathrm{f}$ electrons were established to participate directly in chemical bond formation in $\mathrm{CeO}_{2}$ losing partially their $f$ character. They were found to be localized mostly within the outer valence band. The Ce $5 p$ atomic orbitals were shown to participate in the formation of both the inner and the outer valence molecular orbitals (MOs). A large part in the IVMO formation is taken by the filled $\mathrm{Ce} 5 \mathrm{p}_{1 / 2}, 5 p_{3 / 2}$ and $\mathrm{O} 2 \mathrm{~s}$ atomic shells, while the $\mathrm{Ce} 5 \mathrm{~s}$ electrons participate weakly in the chemical bond formation. The composition and the sequent order of the molecular orbitals in the binding energy range of 0 to $\sim 50 \mathrm{eV}$ were established. A quantitative scheme for the molecular orbitals of $\mathrm{CeO}_{2}$ was built. This scheme is fundamental for understanding the nature of chemical bonding and also for the interpretation of other $\mathrm{X}$-ray spectra of $\mathrm{CeO}_{2}$. Evaluations revealed that the IVMO electrons weaken the chemical bond formed by the OVMO electrons by $37 \%$.
\end{abstract}

\section{Introduction}

Cerium dioxide is known as a non-radioactive structural substitute of actinide oxides $\left(\mathrm{UO}_{2}\right.$ and $\left.\mathrm{PuO}_{2}\right) \cdot{ }^{1,2} \mathrm{CeO}_{2}$-based ceramics are suggested as an inert ${ }^{239} \mathrm{Pu}$ or ${ }^{235} \mathrm{U}$ bearing matrix for nuclear fuel, as well as a matrix for high-level waste disposal. ${ }^{1,3} \mathrm{CeO}_{2}$ is also used an exhaust gas afterburning catalyst $^{4,5}$ and in electronics. ${ }^{6}$

X-ray photoelectron spectroscopy (XPS) has been widely used for the study of the electronic structure of $\mathrm{CeO}_{2} \cdot{ }^{7-11}$ The core and valence XPS spectra of cerium compounds are complicated and structured. This complicates the determination of the oxidation state of cerium on the basis of chemical shifts. ${ }^{7-11}$ Several mechanisms for the formation of the complex $\mathrm{CeO}_{2} \mathrm{XPS}$ structure were considered.

\footnotetext{
${ }^{a}$ Chemistry Department, Lomonosov Moscow State University, Moscow, 119991, Russia

${ }^{b}$ NRC "Kurchatov Institute", Moscow, 123182, Russia

${ }^{c}$ Institute of Solid State Chemistry, Ural Department of RAS, Ekaterinburg, Russia

${ }^{d}$ Department of Earth Sciences, University of Cambridge, Downing Street,

Cambridge, CB2 3EQ, UK. E-mail: apopel@cantab.net; Tel: +44 (0)1223 768357

${ }^{e}$ Department of Materials and London Centre for Nanotechnology,

Imperial College London, London, SW7 2AZ, UK
}

Thus, the authors of ref. 12-16 associated the complex $\mathrm{CeO}_{2}$ XPS structure with the presence of $\mathrm{Ce}^{3+}$ and $\mathrm{Ce}^{4+}$ ions in the samples (mixed valence). However, the authors of ref. 17 suggested that $\mathrm{CeO}_{2}$ contains only $\mathrm{Ce}^{4+}$ ions and on the basis of the calculations they associated the complex Ce 3d XPS structure with the complex final state consisting of the ground final state Ce $3 \mathrm{~d}^{9} 4 \mathrm{f}^{0}$ and the two excited final states $3 \mathrm{~d}^{9} 4 \mathrm{f}^{1} \mathrm{OVMO}^{-1}$ and $3 d^{9} 5 p^{5} n p^{1}$. The unusual final state Ce $3 d^{9} 5 p^{5} n p^{1}$ was attributed to a weakly screened vacancy in the IVMO., ${ }^{9,18}$ These results agree with the experimental core-electron XPS data in ref. 19, which showed that the complex XPS structure appears in the core-electron XPS spectra and does not appear in the valence XPS spectra.

The authors of ref. 18 performed the non-relativistic discretevariational calculations for the $\mathrm{CeO}_{8}$ cluster including a cerium atom and its nearest environment in $\mathrm{CeO}_{2}$ in the $\mathrm{MO}$ binding energy (BE) range of $0-22 \mathrm{eV}$ and qualitatively compared the calculated and experimental spectra. In the present work the valence and the core-level XPS spectra of the single-crystalline $\mathrm{CeO}_{2}$ film on $\mathrm{Si}(001)$ were measured, and the relativistic discretevariational calculations for the $\mathrm{CeO}_{8}$ and $\mathrm{Ce}_{63} \mathrm{O}_{216}$ clusters in the $\mathrm{BE}$ range of 0 to $\sim 50 \mathrm{eV}$ were carried out. A quantitative comparison of the calculated and experimental results was performed. A quantitative scheme of the molecular orbitals for $\mathrm{CeO}_{2}$ was built. 


\section{Experimental}

\subsection{Sample production}

The $\mathrm{CeO}_{2}$ thin film was grown by pulsed laser deposition on a (001) oriented p-doped Si substrate and characterised by XRD and SEM as described in detail in ref. 20. The thin film of $\mathrm{CeO}_{2}$ has nominal thickness $(250 \mathrm{~nm})$. X-ray diffraction analysis suggested that the $\mathrm{CeO}_{2}$ film can be described as a single crystal in a (111) crystallographic orientation.

\subsection{X-ray photoelectron measurements}

The XPS spectra of $\mathrm{CeO}_{2}$ were recorded on a Kratos Axis Ultra DLD spectrometer using monochromatic $\mathrm{Al}-\mathrm{K} \alpha$ radiation $(h \nu=1486.7 \mathrm{eV})$ at a $150 \mathrm{~W}$ X-ray gun power under $1.3 \times 10^{-7} \mathrm{~Pa}$ at room temperature. The analyzed area was an ellipse with minor and major axes of 300 and $700 \mathrm{~nm}$, respectively.

A Kratos charge neutraliser system was used and BEs were measured relatively to the $\mathrm{BE}$ of the $\mathrm{C} 1 \mathrm{~s}$ electrons from hydrocarbons adsorbed on the sample surface, which was found to be equal to $285.0 \mathrm{eV}$. FWHMs are given relatively to that of the C 1s XPS peak for hydrocarbons on the sample surface to be $1.3 \mathrm{eV}^{21}$ The error in the determination of the $\mathrm{BE}$ and the peak width did not exceed $\pm 0.05 \mathrm{eV}$, and the error of the relative peak intensity was $\pm 5 \%$. The inelastically scattered electron-related background was subtracted by the method of Shirley. ${ }^{22}$

Quantitative elemental and ionic analysis was performed for the surface of the studied sample AP1 on the basis of the valence and core-level XPS spectra. The stoichiometric $\left(\mathrm{CeO}_{1.99}\right)$ and ionic $\left(97 \% \mathrm{Ce}^{4+}\right.$ and $\left.3 \% \mathrm{Ce}^{3+}\right)$ compositions of the studied sample were found. More details on the spectral measurements and elemental and ionic analyses are given in ref. 11.

\subsection{Calculations}

The calculations of the electronic structure of $\mathrm{CeO}_{2}\left(R_{\mathrm{Ce}-\mathrm{O}}=\right.$ $0.2344 \mathrm{~nm})^{23}$ were performed for the two types of finite fragments of the crystal lattice: 279-atom $\mathrm{Ce}_{63} \mathrm{O}_{216}$ and small $\mathrm{CeO}_{8}$ clusters. The latter object modeled a cerium atom nearest environment in a dioxide crystal. For the modeling of the boundary conditions for the $\mathrm{Ce}_{63} \mathrm{O}_{216}$ fragment, we used an "extended cluster" scheme described in detail in ref. 24. In this model, the crystal fragment under study consists of two parts: the internal main part (or the "core") and the outer part (or the "shell" of the cluster), the latter part usually includes the atoms of 1-5 coordination spheres surrounding the "core". During the selfconsistency procedure, the electron densities and the potential of atoms in the "shell" are replaced by the corresponding values obtained for the crystallographically equivalent centers of the cluster core. In addition to the introduction of the long-range component of the surrounding crystal potential, the extended cluster is embedded in a pseudopotential of the outer crystal lattice, including several thousand centers with Coulomb and exchange-correlation potentials obtained for the corresponding equivalent atoms in the internal part of the cluster. This pseudopotential modeling of the long-range crystal potential becomes important in the calculations of compounds with noticeable charges on atoms, such as oxides and fluorides.

In the case of $\mathrm{Ce}_{63} \mathrm{O}_{216}$, the core of the cluster included: a Ce atom in the center $\left(\mathrm{Ce}_{1}\right)$ with the eight nearest oxygen neighbors $\left(\mathrm{O}_{1}\right)$ and 12 cerium sites of the next metal coordination sphere with their 48 nearest oxygen atoms. The other atoms of the cluster formed the "shell" and during self-consistency their electron densities and potentials were kept equivalent to those of $\mathrm{Ce}_{1}$ and $\mathrm{O}_{1}$. In the case of small fragment $\mathrm{CeO}_{8}$, the renormalization procedure for the populations of oxygen atoms in valence atomic orbitals (AOs) during self-consistency was used in the calculations. The latter model of the boundary conditions for small clusters also allows one to include into the iterative scheme the stoichiometry of compounds and the possibility of charge redistribution between the outer atoms in the cluster and the surrounding crystal.

In this work, the calculations were carried out using the fully relativistic discrete-variational method (RDV) $)^{25,26}$ with local exchange-correlation potential. ${ }^{27}$ The RDV method is based on the solution of the Dirac-Slater equation for 4-component wave functions transforming according to the irreducible representations of the double point group $\left(D_{4 \mathrm{~h}}\right.$ in the present calculations). For the calculation of symmetry coefficients we used the original code which realizes the projection operator technique $^{25}$ and includes the matrices of the irreducible representations of double point groups obtained in ref. 28 and the transformation matrices presented in ref. 29. The extended basis of 4-component numerical atomic orbitals (AOs) obtained as the solution of the Dirac-Slater equation for the isolated neutral atoms also included the vacant Ce $6 \mathrm{p}_{1 / 2}$ and $6 \mathrm{p}_{3 / 2}$ functions in addition to completely or partially occupied AOs. Numerical Diophantine integration in the calculations of matrix elements was carried out for $698000\left(\mathrm{Ce}_{63} \mathrm{O}_{216}\right)$ and $22000\left(\mathrm{CeO}_{8}\right)$ sample points distributed in the cluster space. This provided the convergence of MO energies within $0.1 \mathrm{eV}$. The calculations of the electronic structure have been proved to be one of the most accurate tools for the determination of the electronic structures of solids. ${ }^{30-32}$

\section{Results and discussion}

The valence XPS structure of $\mathrm{CeO}_{2}(0$ to $\sim 50 \mathrm{eV} \mathrm{BE})$ appears due to OVMO and IVMO formation, as well as due to other processes (Fig. 1). In order to evaluate the contributions of the other processes, the core-level XPS structure was analyzed. For more details see ref. 11. The present work considers only some of the core-level XPS spectra.

\subsection{Core-level XPS structure of $\mathrm{CeO}_{2}$}

As shown in ref. 11, the Ce $3 \mathrm{p}_{1 / 2}, 3 \mathrm{~d}-, 4 \mathrm{~s}-, 4 \mathrm{p}-, 4 \mathrm{~d}-, 5 \mathrm{~s}$ spectra in the $\mathrm{BE}$ range from $\sim 35$ to $\sim 1250 \mathrm{eV}$ exhibit a complex structure due to the many-body perturbation and complex final states. After Ce $3 \mathrm{~d}$ electron photoemission the ground final state Ce $3 \mathrm{~d}^{9} 4 \mathrm{f}^{0}$ and the two excited final states $3 \mathrm{~d}^{9} 4 \mathrm{f}^{1} \mathrm{OVMO}^{-1}$ and $3 d^{9} 5 p^{5} n p^{1}$ appear. The final state Ce $3 d^{9} 5 p^{5} n p^{1}$ was 


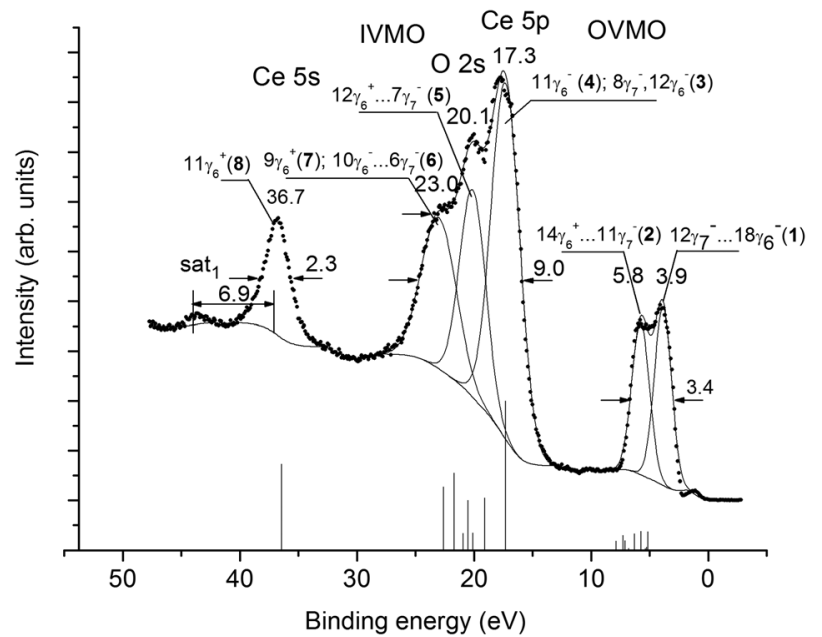

Fig. 1 Valence XPS spectrum of a single-crystalline (111) $\mathrm{CeO}_{2}$ film on the $\mathrm{Si}(001)$ substrate. Vertical bars show the spectrum recorded for the $\mathrm{CeO}_{8}$ cluster.

attributed to a weakly screened vacancy in the IVMO. As a result, the Ce $3 \mathrm{~d}$ XPS spectrum exhibits a complicated structure instead of the expected doublet associated with the spin-orbit splitting $\Delta E_{\mathrm{sl}}(\mathrm{Ce} 3 \mathrm{~d})=18.6 \mathrm{eV}$ (Fig. 2). The same structures are observed in the XPS spectra of other core levels. ${ }^{11}$ However, such a structure is not observed in the spectrum of Ce $5 \mathrm{p}$ electrons (Fig. 1). This confirms the appearance of the $3 \mathrm{~d}^{9} 5 \mathrm{p}^{5} \mathrm{np}^{1}$ final state with a quasi-internal vacancy in the $5 \mathrm{p}$ shell (Fig. 2).

Since the $\mathrm{Ce}^{4+}$ ions do not contain Ce $4 \mathrm{f}$ electrons, the corresponding spectra of the valence electrons do not show the structure associated with multiplet splitting (Fig. 1). As shown in ref. 21, the dynamic effect due to the giant Coster-Kronig transitions is expected in the Ce 4p XPS spectrum. The many-body perturbation $^{11,21}$ is one of the main reasons for the complex

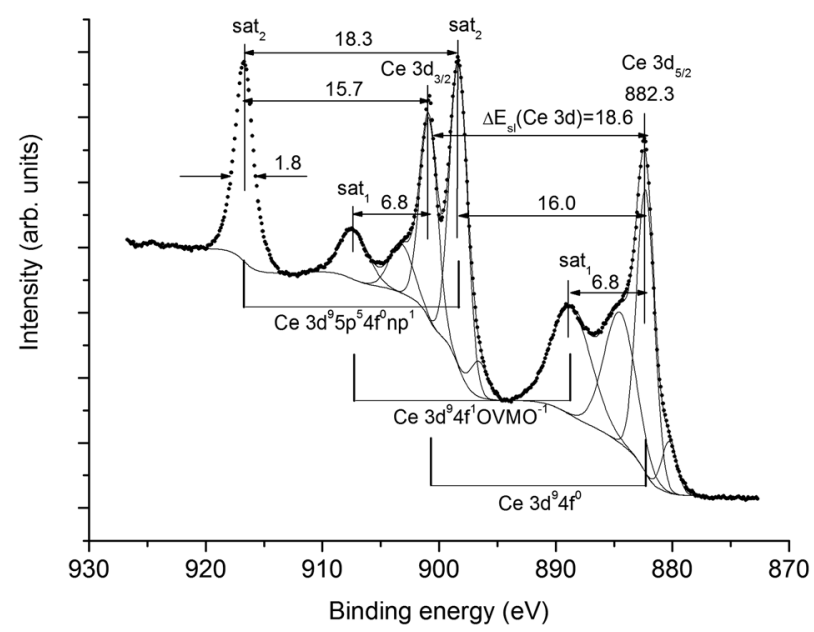

Fig. 2 Ce 3d XPS spectrum of a single-crystalline (111) $\mathrm{CeO}_{2}$ film on the $\mathrm{Si}(001)$ substrate. Structures associated with the ground $\mathrm{Ce} 3 \mathrm{~d}^{9} 4 \mathrm{f}^{0}$ and excited Ce $3 d^{9} 4 f^{1} V M O^{-1}$ and $C e 3 d^{9} 5 p^{5} 4 f^{0} n p^{1}$ final states are shown in the spectrum. structure in the core-level XPS spectra. This structure appears in the Ce $5 \mathrm{~s}$ XPS spectrum, but not in the Ce 5p XPS spectrum. This yields a conclusion that the valence ( 0 to $\sim 35 \mathrm{eV}$ ) XPS structure appears mostly due to the OVMO and IVMO electrons.

The $\mathrm{O} 1 \mathrm{~s}$ spectrum appears as a single peak at $E_{\mathrm{b}}(\mathrm{O} 1 \mathrm{~s})=$ $529.4 \mathrm{eV}$ with FWHM $\Gamma(\mathrm{O}$ 1s) $=1.3 \mathrm{eV}$ (Fig. 3). On a higher BE side from the primary peak a small shoulder at $E_{\mathrm{b}}(\mathrm{O} 1 \mathrm{~s})=$ $531.7 \mathrm{eV}$ can be attributed to hydroxyl groups. The intensity ratio for these peaks is $80 \%\left(\mathrm{CeO}_{2}\right)$ and $20 \%\left(\mathrm{OH}^{-}\right)$. The $\mathrm{O} 2 \mathrm{p}$ and $\mathrm{O} 2 \mathrm{~s}$ features related to the $\mathrm{OH}^{-}$groups increase the error in the XPS intensity measurements (Fig. 1). The BE differences between the valence- and the core-level peaks, as well as the results of cluster calculations were used for the interpretation of valence XPS structures.

\subsection{Electronic structure of the clusters in $\mathrm{CeO}_{2}$}

The ground state electronic configuration of a cerium atom is [Xe] $5 s^{2} 5 p^{6} 4 f^{2} 5 d^{0} 6 s^{2} 6 p^{0}$, where [Xe] is the xenon electronic configuration. The total densities of occupied states (DOS) obtained for the central $\mathrm{Ce}_{1}$ and $\mathrm{O}_{1}$ atoms in the two clusters are shown in Fig. 4. A comparison of the DOS in Fig. 4 shows that the set of discrete molecular levels in the small $\mathrm{CeO}_{8}$ fragment (we have broadened each MO level with a Lorentzian function of a constant width for all MOs) transforms to energy bands of various widths in the large $\mathrm{Ce}_{63} \mathrm{O}_{216}$ cluster. Note that the positions of the centers of gravity of the main bands in both fragments are close to each other. Moreover, the analysis of covalent mixing of Ce $5 \mathrm{p}_{1 / 2}, 5 \mathrm{p}_{3 / 2}$ with $\mathrm{O} 2$ s orbitals and $\mathrm{Ce} 4 \mathrm{f}_{5 / 2}$, $4 \mathrm{f}_{7 / 2}, 5 \mathrm{~d}_{3 / 2}, 5 \mathrm{~d}_{5 / 2}, 6 \mathrm{~s}, 6 \mathrm{p}_{1 / 2}$ and $6 \mathrm{p}_{3 / 2}$ with $\mathrm{O} 2 \mathrm{p}$ AOs shows that the main features of hybridization between metal and oxygen states are also similar in the two clusters. Note that the only visible difference between these spectra is the increase in energy difference between the highest occupied MO (HOMO) and the Ce $4 \mathrm{~s}$ peak $(-37.5 \mathrm{eV})$ in the large cluster by $0.5 \mathrm{eV}$. As seen in Fig. 1, this increase would improve the agreement with experiment. However, since in the large fragment, the energy region under investigation $(-40$ to $0 \mathrm{eV}$ ) contains 2231 orbitals,

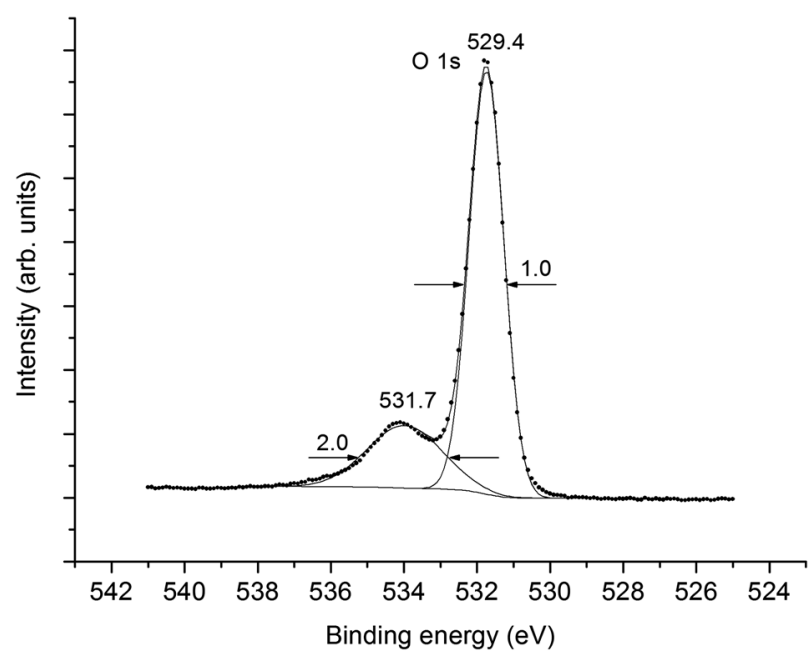

Fig. 3 An XPS narrow-scan of $\mathrm{O}$ 1s of the single-crystalline $\mathrm{CeO}_{2}$ film. 


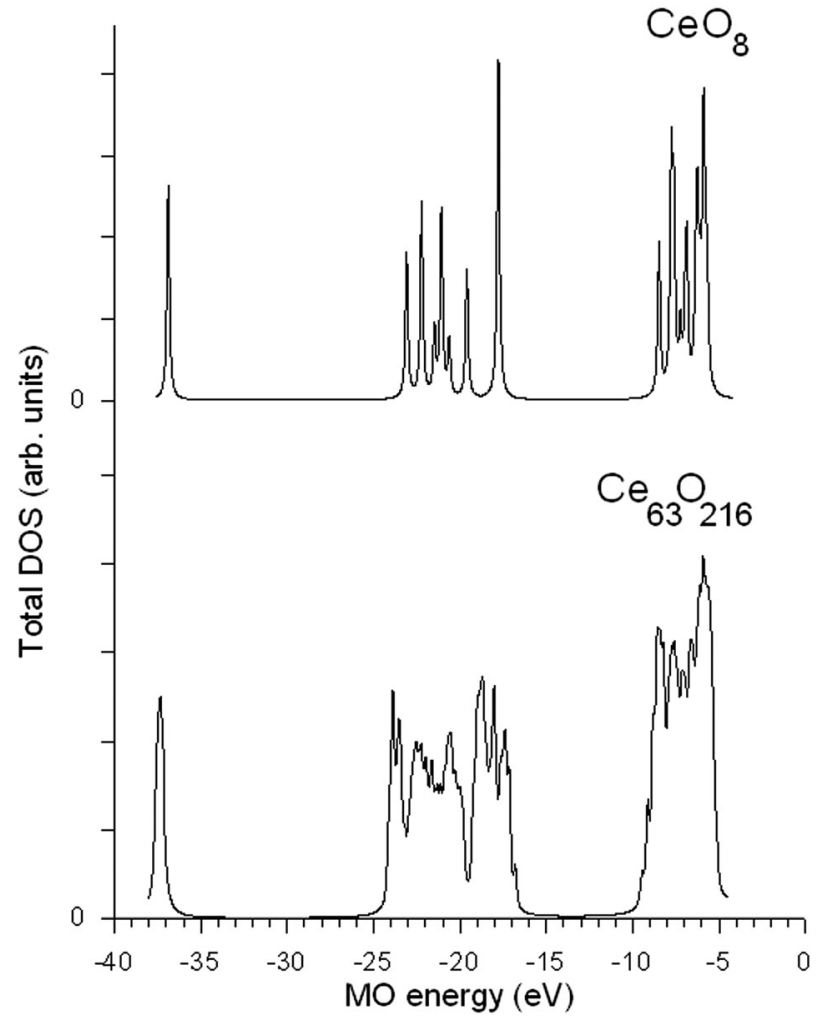

Fig. 4 Total DOS for the central part of $\mathrm{CeO}_{8}$ and $\mathrm{Ce}_{63} \mathrm{O}_{216}$ clusters.

for the illustration of the general structure of the main molecular states, we use the results of RDV calculations for the small $\mathrm{CeO}_{8}$ cluster. It is worth mentioning that in Fig. 1, the theoretical MO intensities were calculated using photoionization crosssections. ${ }^{33,34}$ The characteristics of all vacant and occupied MOs in the energy region from -40 to $+15 \mathrm{eV}$ obtained for this cluster are given in Table 1. The MO LCAO (Molecular Orbitals as Linear Combinations of Atomic Orbitals) approach was employed in order to consider the chemical bond in terms of atomic and molecular shells.

The IVMO and the OVMO formation is a result of the hybridization of Ce and $\mathrm{O}$ orbitals (Ce $5 \mathrm{~s}, 5 \mathrm{p}, 4 \mathrm{f}$, $6 \mathrm{~s}$ and $\mathrm{O} 2 \mathrm{~s}$, $2 \mathrm{p}$ and empty Ce $5 \mathrm{~d}, 6 \mathrm{p}$ AOs). Unlike the results of nonrelativistic calculations for $\mathrm{CeO}_{2}$ showing a significant participation of Ce $5 \mathrm{~S}$ AO in MO formation, ${ }^{18}$ the relativistic calculations show that Ce $5 \mathrm{~s}$ states do not hybridize with oxygen orbitals (Table 1). The Ce $6 \mathrm{~s}$ and $6 \mathrm{p}$ AOs take a notable part in the formation of vacant MOs. The Ce $4 \mathrm{f}$ AOs mostly participate in OVMO formation, while the Ce $5 \mathrm{p}, 5 \mathrm{~d}$ AOs participate in both the OVMO and the IVMO. Especially a high mixing of the Ce $5 p_{3 / 2}$ and the $\mathrm{O} 2 \mathrm{~s}$ AOs was observed for the $12 \gamma_{6}{ }^{-}, 8 \gamma_{7}{ }^{-}$(3) and $10 \gamma_{6}{ }^{-}, 6 \gamma_{7}{ }^{-}$(6) IVMOs (Table 1). The hybridization of the Ce $5 p_{1 / 2}$ and $O 2 s$ AOs in the $11 \gamma_{6}{ }^{-}(4)$ and $9 \gamma_{6}{ }^{-}$(7) IVMOs is even higher due to a decrease in the BE difference between Ce $5 p_{1 / 2}$ and $O 2$ s levels (Fig. 1). These results together with the photoionization cross-sections ${ }^{33,34}$ allow one to build a theoretical XPS spectrum (Table 2) and to interpret the corresponding experimental valence XPS spectral structure of $\mathrm{CeO}_{2}$ (Fig. 1).

\subsection{Valence XPS spectrum of $\mathrm{CeO}_{2}$}

The valence XPS spectrum exhibits several explicit features. This BE range can be subdivided into components (Fig. 1). Although formalistic, this subdivision allows one to compare the theoretical and experimental spectra of $\mathrm{CeO}_{2}$ (Fig. 1 and Table 2).

The experimental spectrum reflects the excited electronic state with a hole in a level, while the theoretical spectrum corresponds to the ground state. Therefore, the BE values calculated for the transition state should be used. ${ }^{35}$ However, it is known that the valence BEs calculated for the transition state usually differ from those for the ground state by a constant shift toward a higher value. Therefore, the BEs (Table 1) are increased by $5.14 \mathrm{eV}$ (Table 2). With the MO composition (Table 1) and photoionization cross-sections in mind, ${ }^{33,34}$ the intensities of the spectral ranges were calculated (Table 2). It has to be taken into account that $\mathrm{CeO}_{2}$ XPS reflects the band structure and consists of bands widened due to the solid-state effects. Despite the approximation imperfection, a satisfactory quantitative agreement between the theoretical and experimental data was observed (Fig. 1 and Table 2).

The OVMO band can be subdivided into two components $(1,2)$ at 3.9 and $5.8 \mathrm{eV}$ (Fig. 1 and Table 2). Component (1) is mostly due to the Ce $4 \mathrm{f}$ and $\mathrm{O} 2 \mathrm{p}$ electrons, while component (2) is due to the Ce $5 \mathrm{~d}$ and $\mathrm{O} 2 \mathrm{p}$ electrons (Table 1 ). This agrees with the results of non-relativistic calculations for the $\mathrm{CeO}_{8}$ cluster $^{18}$ and with the resonant emission (OVMO $\rightarrow$ Ce $4 \mathrm{~d}^{9}$ ) spectroscopy data ${ }^{36}$ for $\mathrm{CeO}_{2}$. Under the resonant excitation at $124.8 \mathrm{eV}$, component (1) grows considerably at $3.56 \mathrm{eV}$. This confirms that this peak reflects the $\mathrm{Ce} 4 \mathrm{f}$ electronic states. The resonant emission spectrum of $\mathrm{CeO}_{2}$ does not exhibit a feature at a lower energy side from the OVMO band associated with the Ce $4 \mathrm{f}$ electrons, whereas the corresponding spectrum of $\mathrm{Ce}_{2} \mathrm{O}_{3}$ exhibits this feature at $1.95 \mathrm{eV}$. This indicates that a stoichiometric $\mathrm{CeO}_{2}$ consists of only $\mathrm{Ce}^{4+}$ ions. Thus, the Ce $4 \mathrm{f}$ related feature at $1.1 \mathrm{eV}$ in the spectrum of $\mathrm{CeO}_{2}$ (Fig. 1) appears due to $\mathrm{Ce}^{3+}$ ions formed on the surface under the $\mathrm{Al} \mathrm{K}_{\alpha} \mathrm{X}$-ray beam. In previous papers, the increase in the $\mathrm{Ce}^{3+}$ concentration on the surface of $\mathrm{CeO}_{2}$ under the $\mathrm{Al} \mathrm{K} \mathrm{K}_{\alpha} \mathrm{X}$-ray beam ${ }^{36,37}$ and increased temperature $\mathrm{f}^{5,38,39}$ were observed.

The IVMO spectrum can be formally subdivided into several ranges (Fig. 1 and Table 2). The Ce 5 s spectrum does not show a $40 \%$ high-BE satellite with $\Delta E_{\mathrm{sat} 2}=15 \mathrm{eV}$, the intensity of which was taken into account for the determination of the experimental intensity $(15 \%)$ of the $11 \gamma_{6}{ }^{+}$IVMO (Table 2). The calculated $\left(\Gamma_{\mathrm{T}}(\mathrm{OVMO})=2.72 \mathrm{eV}, I_{\mathrm{T}}(\mathrm{OVMO})=\right.$ $18 \%$ and $\left.I_{\mathrm{T}}(\mathrm{IVMO})=82 \%\right)$ and the experimental $\left(\Gamma_{\mathrm{E}}(\mathrm{OVMO})=\right.$ $3.4 \mathrm{eV}, I_{\mathrm{E}}(\mathrm{OVMO})=22 \%$ and $\left.I_{\mathrm{E}}(\mathrm{IVMO})=79 \%\right)$ data are comparable (Table 2 and Fig. 1). A satisfactory agreement between the theoretical and experimental BEs of some electronic shells was reached (Table 2). As noted, the nonrelativistic calculations ${ }^{18}$ allowed only a qualitative interpretation of the low-BE part of the valence XPS spectrum of $\mathrm{CeO}_{2}$. The results of the present relativistic calculations allow a 
Table 1 MO composition, energy $E_{0}{ }^{a}(\mathrm{eV})$ and the photoionization cross-sections $\sigma_{\mathrm{i}}^{b}$ for the $\mathrm{CeO}_{8}$ cluster

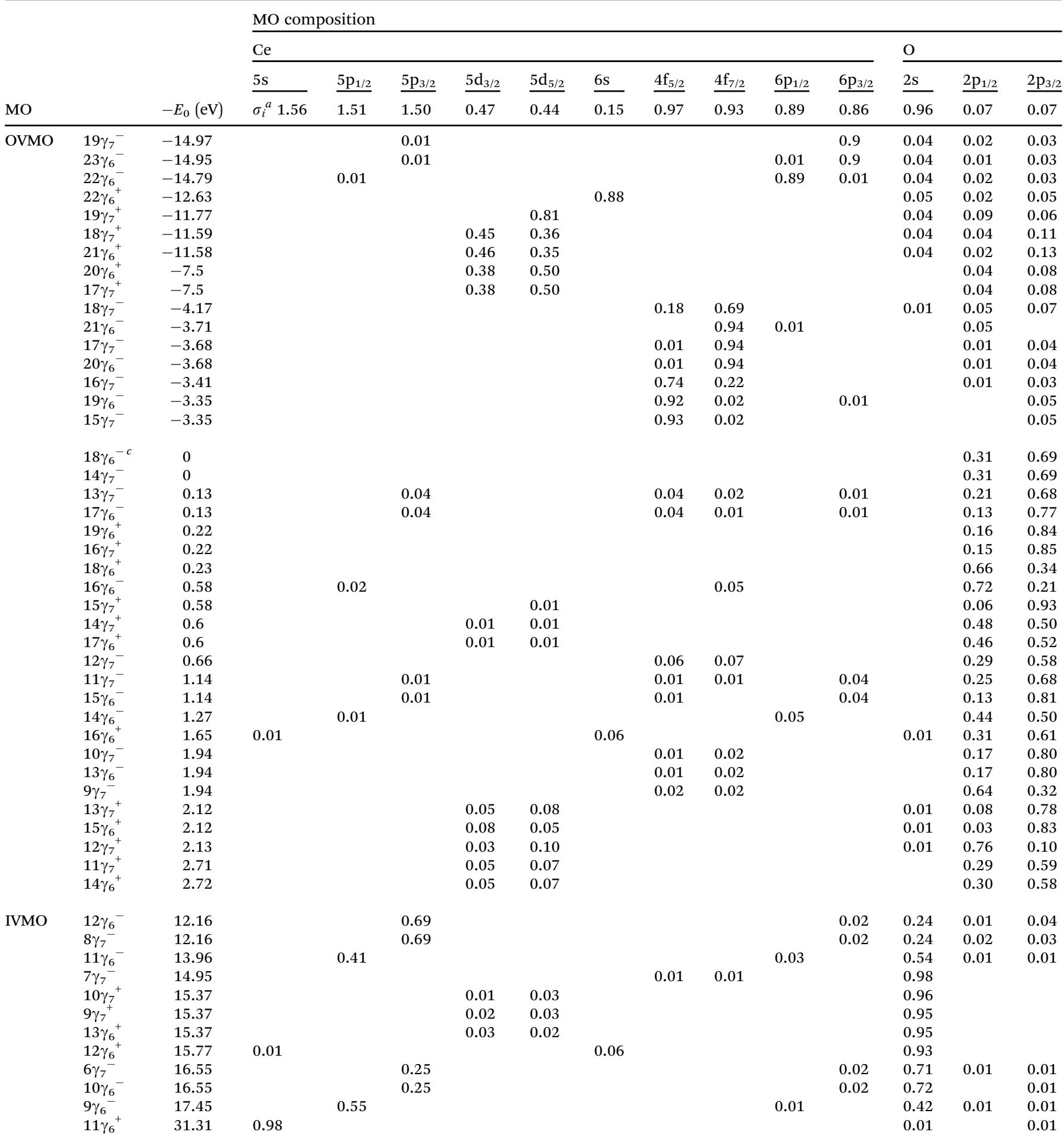

${ }^{a}$ Levels shifted by $13.01 \mathrm{eV}$ toward the positive values (upward). ${ }^{b}$ Photoionization cross-sections $\sigma_{\mathrm{i}}$ (kiloBarn per electron) from ref. 33. ${ }^{c}$ Highest occupied MO (HOMO; 2 electrons), the occupation number for all the orbitals is 2 .

satisfactory quantitative identification of the $\mathrm{CeO}_{2}$ valence XPS spectrum in the $\mathrm{BE}$ range from 0 to $\sim 50 \mathrm{eV}$ (Table 2 and Fig. 1). These data will promote the development of the RDV method and help to understand the chemical bond nature and to construct a quantitative $\mathrm{MO}$ scheme in $\mathrm{CeO}_{2}$.

\subsection{Molecular orbital scheme of $\mathrm{CeO}_{2}$}

With the BE differences between the valence- and the core-level peaks, as well as the calculation results for atomic ${ }^{34} \mathrm{Ce}$ and the $\mathrm{CeO}_{8}$ cluster, a quantitative scheme of the molecular orbitals for $\mathrm{CeO}_{2}$ was built (Fig. 5). In the accepted approximation one 
Table 2 Parameters of $\mathrm{CeO}_{2}$ XPS spectra, $\mathrm{CeO}_{8}$ cluster and Ce $5 \mathrm{p}$ and $\mathrm{Ce} 4 \mathrm{f} \mathrm{DOS} \rho_{\mathrm{i}}\left(\mathrm{e}^{-}\right)$

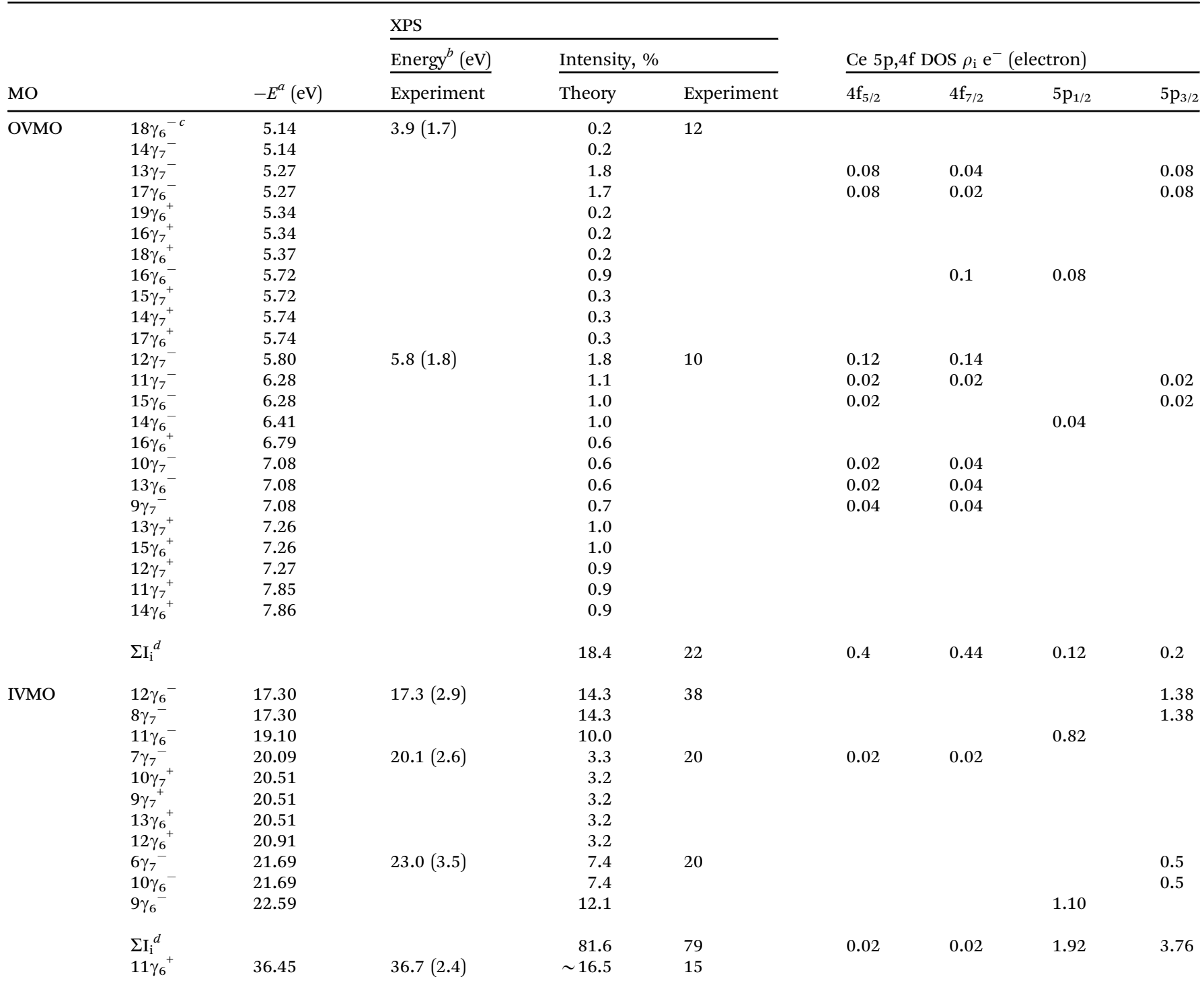

${ }^{a}$ Energies shifted toward higher values (down) by $5.14 \mathrm{eV} .{ }^{b}$ FWHMs are given in the parentheses in eV. ${ }^{c}$ The highest occupied MO $(2$ electrons), the occupation number for other orbitals is $2 .{ }^{d}$ Total intensities and Ce $5 \mathrm{p}, 4 \mathrm{f}$ DOS.

can formally separate the antibonding $12 \gamma_{6}{ }^{-}, 8 \gamma_{7}{ }^{-}$(3) and $11 \gamma_{6}{ }^{-}$(4) and the corresponding bonding $10 \gamma_{6}{ }^{-}, 6 \gamma_{7}$ (6) and $9 \gamma_{6}{ }^{-}$(7) IVMOs, as well as the quasiatomic $7 \gamma_{7}{ }^{-}, 10 \gamma_{7}{ }^{+}, 9 \gamma_{7}{ }^{+}$, $13 \gamma_{6}{ }^{+}$and $12 \gamma_{6}{ }^{+}$(5) IVMOs associated mostly with the $\mathrm{O} 2 \mathrm{~s}$ states which have to be close in magnitude.

Indeed, the $\mathrm{O} 1 \mathrm{~s}$ spectrum of $\mathrm{CeO}_{2}$ allows one to evaluate that the inequality of $\mathrm{O} 2 \mathrm{~s}$ states must not exceed $1 \mathrm{eV}$, since the O 1s peak was observed to be $\operatorname{sharp}(\Gamma=1.0 \mathrm{eV})$ and symmetric (Fig. 3). The $\mathrm{O} 2 \mathrm{~s} \mathrm{BE}$ must be $E_{\mathrm{b}}(\mathrm{O} 2 \mathrm{~s})=21.1 \mathrm{eV}$, since for atomic oxygen $\Delta E_{\mathrm{O}}=E_{\mathrm{b}}(\mathrm{O} 1 \mathrm{~s})-E_{\mathrm{b}}(\mathrm{O} 2 \mathrm{~s})=508.3 \mathrm{eV}^{34}$ and the $\mathrm{O} 1 \mathrm{~s}$ BE is $E_{\mathrm{b}}=529.4 \mathrm{eV}$ (Fig. 3). These data partially agree with the results of theoretical calculations (Table 2). Taking into account that the calculated BE difference for Ce atoms is $\Delta E_{\mathrm{Ce}}^{\mathrm{T}}=864.5 \mathrm{eV}$ $\left(E_{\mathrm{b}}\left(\mathrm{Ce} 3 \mathrm{~d}_{5 / 2}\right)=883.3 \mathrm{eV}\right.$ and $\left.E_{\mathrm{b}}\left(\mathrm{Ce} 5 \mathrm{p}_{3 / 2}\right)=18.8 \mathrm{eV}\right),{ }^{34}$ while the difference between the Ce $3 \mathrm{~d}_{5 / 2}$ and $12 \gamma_{6}{ }^{-}, 8 \gamma_{7}{ }^{-}$(3) IVMO is $\Delta E_{1}=865.0 \mathrm{eV}$, one can find that $\Delta_{1}=0.5 \mathrm{eV}$ (Fig. 5).
This confirms the antibonding nature of the $12 \gamma_{6}{ }^{-}, 8 \gamma_{7}{ }^{-}$(3) IVMO. The FWHM of the IVMO XPS features does not allow to arrive at a conclusion on their bonding or antibonding nature. However, one can suggest that these orbitals partially lose their antibonding nature (Table 2 and Fig. 5, see also ref. 40) due to the contribution of $5 \%$ of the $\mathrm{O} 2 \mathrm{p}$ and $2 \%$ of the Ce $6 \mathrm{p}$ AO in the $12 \gamma_{6}{ }^{-}, 8 \gamma_{7}{ }^{-}$(3) IVMO.

\subsection{Contribution of valence electrons to the chemical bond in $\mathrm{CeO}_{2}$}

In this work, we used the values of overlap populations $\left(n_{\mathrm{ij}}\right)$ of various pairs of metal and oxygen $\mathrm{AOs}^{41}$ to evaluate their contributions to the chemical bond. Table 3 shows these values for the outer and inner valence electrons obtained in the non-relativistic (NDV) and relativistic (RDV) calculations. Positive and negative values correspond to the bonding and 


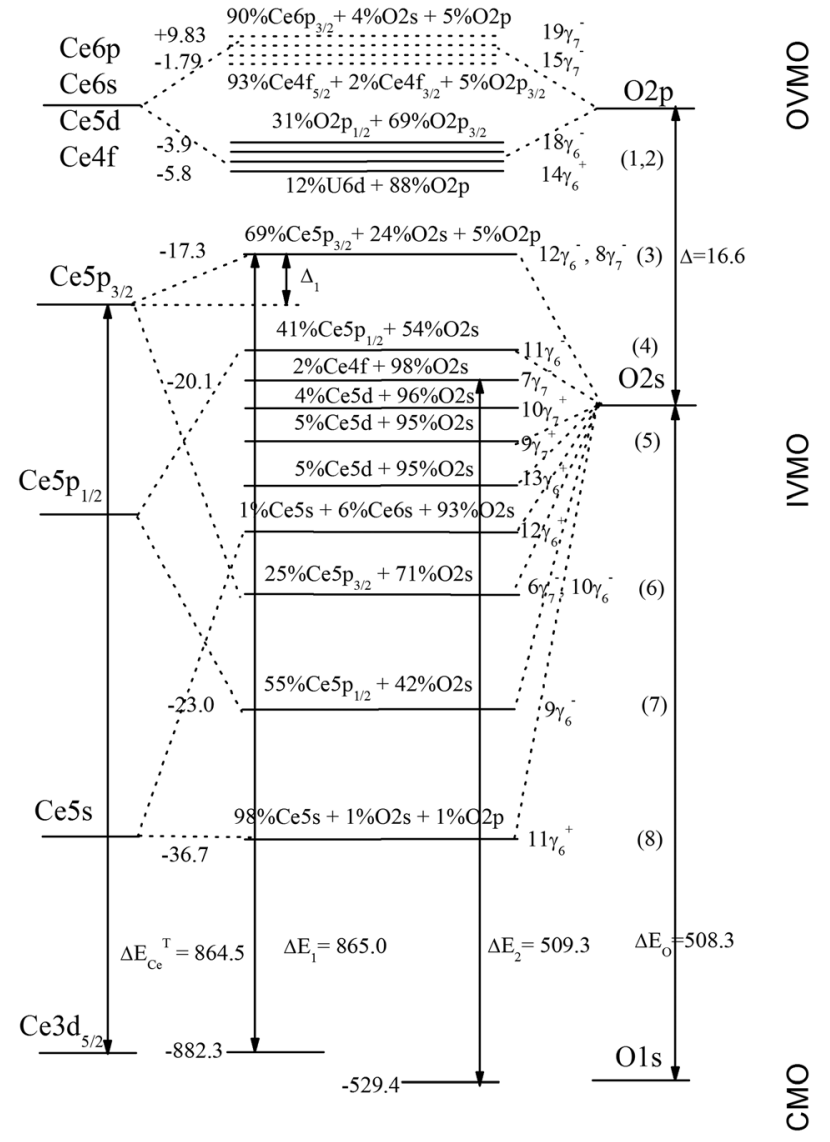

$\mathrm{AO}$

$\mathrm{MO}$

$\mathrm{AO}$

Fig. 5 Molecular orbital scheme for the $\mathrm{CeO}_{8}$ cluster built on the basis of theoretical and experimental data. Chemical shift is not shown. Arrows indicate experimentally measured BE differences. Experimental BEs (eV) are shown on the left. The energy scale is not shown.

Table 3 Overlap populations for $\mathrm{CeO}_{2}$ (per one ligand, $\times 10^{3}$ ), obtained in the relativistic (RDV) and non-relativistic (NDV) calculations of the $\mathrm{CeO}_{8}$ cluster

\begin{tabular}{|c|c|c|c|}
\hline \multirow[b]{2}{*}{ Bonds in $\mathrm{CeO}_{2}$} & \multicolumn{2}{|l|}{$\mathrm{RDV}^{a}$} & \multirow[b]{2}{*}{ NDV } \\
\hline & Partial & Total & \\
\hline Ce $4 \mathrm{f}_{5 / 2}-\mathrm{O} 2 \mathrm{p}_{1 / 2} ; \mathrm{O} 2 \mathrm{p}_{3 / 2}$ & $1 ; 21$ & 49 & 43 \\
\hline Ce $4 \mathrm{f}_{7 / 2}-\mathrm{O} 2 \mathrm{p}_{1 / 2} ; \mathrm{O} 2 \mathrm{p}_{3 / 2}$ & $15 ; 12$ & & \\
\hline Ce $4 f_{5 / 2}-O 2 s$ & 3 & & \\
\hline Ce $4 f_{7 / 2}-O 2 s$ & 4 & 7 & 5 \\
\hline Ce $6 p_{1 / 2}-O 2 p_{1 / 2} ; O 2 p_{3 / 2}$ & $18 ; 5$ & & \\
\hline Ce $6 \mathrm{p}_{3 / 2}-\mathrm{O} 2 \mathrm{p}_{1 / 2} ; \mathrm{O} 2 \mathrm{p}_{3 / 2}$ & $3 ; 42$ & 68 & 67 \\
\hline Ce $6 p_{1 / 2}-O 2 s$ & 11 & & \\
\hline Ce $6 \mathrm{p}_{3 / 2}-\mathrm{O} 2 \mathrm{~s}$ & 19 & 30 & 27 \\
\hline Ce $6 s-O 2 p_{1 / 2} ; O 2 p_{3 / 2}$ & $4 ; 7$ & 11 & 5 \\
\hline $\mathrm{Ce} 6 \mathrm{~s}-\mathrm{O} 2 \mathrm{~s}$ & 26 & 26 & 27 \\
\hline Ce $5 \mathrm{~d}_{3 / 2}-\mathrm{O} 2 \mathrm{p}_{1 / 2} ; \mathrm{O} 2 \mathrm{p}_{3 / 2}$ & $4 ; 72$ & & \\
\hline Ce $5 \mathrm{~d}_{5 / 2}-\mathrm{O} 2 \mathrm{p}_{1 / 2} ;$ O $2 \mathrm{p}_{3 / 2}$ & $58 ; 55$ & 189 & 179 \\
\hline Ce $5 d_{3 / 2}-O 2 s$ & 12 & & \\
\hline Ce $5 d_{5 / 2}-O 2 s$ & 19 & 31 & 30 \\
\hline$\Sigma_{\text {OVMO }}^{b}$ & 411 & 411 & 383 \\
\hline Ce $5 p_{1 / 2}-O 2 p_{1 / 2} ; O 2 p_{3 / 2}$ & $-8 ;-18$ & & \\
\hline Ce $5 \mathrm{p}_{3 / 2}-\mathrm{O} 2 \mathrm{p}_{1 / 2} ; \mathrm{O} 2 \mathrm{p}_{3 / 2}$ & $-23 ;-40$ & -89 & -91 \\
\hline Ce $5 p_{1 / 2}-O 2 s$ & -7 & & \\
\hline Ce $5 p_{3 / 2}-O 2 s$ & -21 & -28 & -31 \\
\hline Ce $5 \mathrm{~s}-\mathrm{O} 2 \mathrm{p}_{1 / 2} ; \mathrm{O} 2 \mathrm{p}_{3 / 2}$ & $-10 ;-20$ & -30 & -37 \\
\hline $\mathrm{Ce} 5 \mathrm{~s}-\mathrm{O} 2 \mathrm{~s}$ & -3 & -3 & -5 \\
\hline$\Sigma_{\text {IVMO }}^{b}$ & -150 & -150 & -164 \\
\hline
\end{tabular}

${ }^{a}$ Partial and total contribution. ${ }^{b}$ Total OVMO and IVMO contribution. antibonding interactions, respectively. The relativistic and nonrelativistic results differ slightly. For example, the contribution from the Ce $4 \mathrm{f}-\mathrm{O} 2 \mathrm{p}$, Ce $6 \mathrm{~s}-\mathrm{O} 2 \mathrm{p}$ and $\mathrm{Ce} 5 \mathrm{~d}-\mathrm{O} 2 \mathrm{p}$ bonds increases in the relativistic approach, which reveals the bonding nature of these states. The Ce $5 \mathrm{~s}-\mathrm{O} 2 \mathrm{~s}, 2 \mathrm{p}$ interactions are less antibonding in the non-relativistic calculation than in the relativistic one. This can be explained by the fact that in the relativistic calculation, the Ce $5 \mathrm{~s} \mathrm{BE}$ increases significantly compared to that calculated in the non-relativistic approach.

The contribution to bonding from the outer valence shells in $\mathrm{CeO}_{2}$ is (411) (Table 3). The largest bonding contributions come from the Ce $4 \mathrm{f}-\mathrm{O} 2 \mathrm{p}$ (49), Ce 6p-O 2p (68) and Ce 5d-O 2p (189) AOs. The inner valence electrons of Ce provide the total antibonding contribution of -150 . The highest antibonding contribution is obtained from the Ce $5 \mathrm{p}-\mathrm{O} 2 \mathrm{p}(-89)$ states. To summarize, the IVMO electrons weaken the chemical bond by $37 \%$. The total contribution of the valence electrons to the chemical bond in $\mathrm{CeO}_{2}$ is 261 .

\section{Conclusions}

On the basis of the relativistic cluster calculations, a quantitative interpretation of the outer ( 0 to $\sim 15 \mathrm{eV} \mathrm{BE}$ ) and inner $(\sim 15$ to $\sim 50 \mathrm{eV} \mathrm{BE})$ valence XPS spectral structures of $\mathrm{CeO}_{2}$ was performed.

It was theoretically shown and experimentally confirmed that the Ce $4 \mathrm{f}$ electrons ( 0.86 of the $\mathrm{Ce} 4 \mathrm{f}$ electron) participate directly in chemical bond formation in $\mathrm{CeO}_{2}$ partially losing the $\mathrm{f}$-character. The contributions from Ce $4 \mathrm{f}$ orbitals are located mainly within the outer valence band.

It was found that the Ce $5 p$ electrons not only participate effectively (experimentally measurable) in the formation of the IVMO, but also participate noticeably in the formation of the occupied OVMO ( 0.32 of the Ce $5 p$ electron). The largest part in the IVMO formation is taken by the Ce $5 p_{1 / 2}, 5 p_{3 / 2}$ and the $O 2 \mathrm{~s}$ AOs of the neighboring cerium and oxygen. The Ce $5 \mathrm{~s}$ electrons participate weakly in the chemical bond formation.

The composition and the sequent order of the molecular orbitals in the binding energy range from 0 to $\sim 50 \mathrm{eV}$ were established. A quantitative scheme of the molecular orbitals for $\mathrm{CeO}_{2}$ was built. This scheme is fundamental for understanding the nature of chemical bonding and for the interpretation of other X-ray spectra of $\mathrm{CeO}_{2}$. The evaluation of the contribution from various electrons to the chemical bond revealed that the antibonding contribution from IVMO electrons is near $37 \%$ of the chemical bond formed by the OVMO electrons.

\section{Conflicts of interest}

There are no conflicts to declare.

\section{Acknowledgements}

The work was supported by the RFBR grant no. 17-03-00277a. The authors acknowledge support from Lomonosov Moscow 
State University Program of Development for providing access to the XPS facility. M. V. Ryzhkov acknowledges financial support of FASO of Russia ISSC of the Ural Branch of RAS No. AAAA-A16-116122810214-9. A. J. Popel acknowledges funding from the UK EPSRC (grant EP/I036400/1) and Radioactive Waste Management Ltd (formerly the Radioactive Waste Management Directorate of the UK Nuclear Decommissioning Authority, contract NPO004411A-EPS02), a maintenance grant from the Russian Foundation for Basic Research (projects 13-03-90916) and CSAR bursary.

\section{References}

1 P. D. Edmondson, Y. Zhang, S. Moll, F. Namavar and W. J. Weber, Irradiation effects on microstructure change in nanocrystalline ceria - Phase, lattice stress, grain size and boundaries, Acta Mater., 2012, 60, 5408-5416.

2 M. Kinoshita, K. Yasunaga, T. Sonoda, A. Iwase, N. Ishikawa, M. Sataka, K. Yasuda, S. Matsumura, H. Y. Geng, T. Ichinomiya, Y. Chen, Y. Kaneta, M. Iwasawa, T. Ohnuma, Y. Nishiura, J. Nakamura and H. Matzke, Recovery and restructuring induced by fission energy ions in high burnup nuclear fuel, Nucl. Instrum. Methods Phys. Res., Sect. B, 2009, 267, 960-963.

3 B. E. Burakov, M. I. Ojovan and W. E. Lee, Crystalline Materials for Actinide Immobilisation, in Materials for Engineering, ed. W. E. Lee, Imperial College Press, London, 2011, vol. 1, pp. 58-59.

4 D. R. Mullins, S. H. Overbury and D. R. Huntley, Electron spectroscopy of single crystal and polycrystalline cerium oxide surfaces, Surf. Sci., 1998, 409, 307-319.

5 V. Stetsovych, F. Pagliuca, F. Dvorak, T. Duchon, M. Vorokhta, M. Aulicka, J. Lachnitt, S. Schernich, I. Matolinova, K. Veltruska, T. Skala, D. Mazur, J. Myslivecek, J. Libuda and V. Matolin, Epitaxial Cubic $\mathrm{Ce}_{2} \mathrm{O}_{3}$ Films via Ce- $\mathrm{CeO}_{2}$ Interfacial Reaction, J. Phys. Chem. Lett., 2013, 4, 866-871.

6 Y. Zhu, N. Jain, M. K. Hudait, D. Maurya, R. Varghese and S. Priya, X-ray photoelectron spectroscopy analysis and band offset determination of $\mathrm{CeO}_{2}$ deposited on epitaxial (100), (110), and (111)Ge, J. Vac. Sci. Technol., B, 2014, 32, 011217.

7 P. Burroughs, A. Hamnett, A. F. Orchard and G. Thornton, Satellite structure in the X-ray photoelectron spectra of some binary and mixed oxides of lanthanum and cerium, J. Chem. Soc., Dalton Trans., 1976, 1686-1698.

8 Yu. A. Teterin and A. S. Baev, Rentgenovskaya Fotoelektronnaya Spektroskopiya Soedinenii Lantanoidov (X-ray Photoelectron Spectroscopy of Lanthanide Compounds), TsNII atominform, Moscow, 1987 (in Russian).

$9 \mathrm{Yu}$. A. Teterin and A. Yu. Teterin, Structure of X-ray photoelectron spectra of lanthanide compound, Russ. Chem. Rev., 2002, 71, 347-381.

10 Yu. A. Teterin, S. V. Stefanovskīi, S. V. Yudintsev, G. N. Bek-Uzarov, A. Yu. Teterin, K. I. Maslakov and I. O. Utkin, $\mathrm{X}$-ray photoelectron study of calcium cerium titanate ceramics, Russ. J. Inorg. Chem., 2004, 49, 87-94.
11 K. I. Maslakov, Yu. A. Teterin, A. J. Popel, A. Yu. Teterin, K. E. Ivanov, S. N. Kalmykov, V. G. Petrov, P. K. Petrov and I. Farnan, XPS study of ion irradiated and unirradiated $\mathrm{CeO}_{2}$ bulk and thin film samples, Appl. Surf. Sci., 2018, 448, 154-162.

12 A. Kotani, T. Jo and J. C. Parlebas, Many-body effects in core-level spectroscopy of rare-earth compounds, Adv. Phys., 1988, 37, 37-85.

13 A. Kotani and H. Ogasawara, Theory of core-level spectroscopy of rare-earth oxides, J. Electron Spectrosc. Relat. Phenom., 1992, 60, 257-299.

14 A. Fujimori, Mixed-valent ground state of $\mathrm{CeO}_{2}$, Phys. Rev. B: Condens. Matter Mater. Phys., 1983, 28, 2281-2283.

15 A. Kotani, Theory of core-level spectroscopy in $\mathrm{f}$ and $\mathrm{d}$ electron systems, J. Electron Spectrosc. Relat. Phenom., 1999, 100, 75-104.

16 E. Shoko, M. F. Smith and R. H. McKenzie, Mixed valency in cerium oxide crystallographic phases: Valence of different cerium sites by the bond valence method, Phys. Rev. B: Condens. Matter Mater. Phys., 2009, 79, 134108.

17 G. Thornton and M. J. Dempsey, Final-state effects in the $3 \mathrm{~d}$ and $4 \mathrm{~d}$ X-ray photoelectron spectra of $\mathrm{CeO}_{2}$, Chem. Phys. Lett., 1981, 77, 409-412.

18 M. V. Ryzhkov, V. A. Gubanov, Yu. A. Teterin and A. S. Baev, Electronic structure, chemical bonding and X-ray photoelectron spectra of light rare-earth oxides, Z. Phys. B: Condens. Matter, 1985, 59, 1-6.

19 Yu. A. Teterin, A. Yu. Teterin, A. M. Lebedev and I. O. Utkin, The XPS spectra of cerium compounds containing oxygen, J. Electron Spectrosc. Relat. Phenom., 1998, 88-91, 275-279.

20 A. J. Popel, S. Le Solliec, G. I. Lampronti, J. Day, P. K. Petrov and I. Farnan, The effect of fission-energy Xe ion irradiation on the structural integrity and dissolution of the $\mathrm{CeO}_{2}$ matrix, J. Nucl. Mater., 2017, 484, 332-338.

$21 \mathrm{Yu}$. A. Teterin and A. Yu, Teterin, The structure of X-ray photoelectron spectra of light actinide compounds, Russ. Chem. Rev., 2004, 73, 541-580.

22 D. Shirley, High-Resolution X-ray Photoemission Spectrum of the Valence Bands of Gold, Phys. Rev. B: Solid State, 1972, 5, 4709-4714.

23 M. Wolcyrz and L. Kepinski, Rietveld refinement of the structure of CeOCI formed in $\mathrm{Pd} / \mathrm{CeO}_{2}$ catalyst: Notes on the existence of a stabilized tetragonal phase of $\mathrm{La}_{2} \mathrm{O}_{3}$ in LaPdO system, J. Solid State Chem., 1992, 99, 409-413.

24 M. V. Ryzhkov, N. I. Medvedeva and V. A. Gubanov, Substitution of $\mathrm{Pb}$ by $\mathrm{Ag}$ as a way to obtain new high temperature superconductors: A quantum chemical point of view, J. Phys. Chem. Solids, 1995, 56, 1231-1237.

25 A. Rosen and D. E. Ellis, Relativistic molecular calculations in the Dirac-Slater model, J. Chem. Phys., 1975, 62, 3039-3049.

26 D. E. Ellis and G. L. Goodman, Self-consistent Dirac-Slater calculations for molecules and embedded clusters, Int. J. Quantum Chem., 1984, 25, 185-200.

27 O. Gunnarsson and B. I. Lundqvist, Exchange and correlation in atoms, molecules, and solids by the spin-density-functional formalism, Phys. Rev. B: Solid State, 1976, 13, 4274-4298. 
28 P. Pyykkö and H. Toivonen, Tables of Representation and Rotation Matrices for the Relativistic Irreducible Representations of 38 Point Groups, Acta Acad. Abo., Ser. B, 1983, 43, 1-50.

29 D. A. Varshalovich, A. N. Moskalev and V. K. Khersonskii, Quantum Theory of Angular Momentum, World Scientific, 1988.

30 A. H. Reshak, Thermoelectric properties for AA- and AB-stacking of a carbon nitride polymorph $\left(\mathrm{C}_{3} \mathrm{~N}_{4}\right), R S C A d v ., 2014,4$, 63137-63142.

31 G. E. Davydyuk, O. Y. Khyzhun, A. H. Reshak, H. Kamarudin, G. L. Myronchuk, S. P. Danylchuk, A. O. Fedorchuk, L. V. Piskach, M. Y. Mozolyuk and O. V. Parasyuk, Photoelectrical properties and the electronic structure of $\mathrm{Tl}_{1-x} \mathrm{In}_{1-x} \mathrm{Sn}_{x} \mathrm{Se}_{2}$ $(x=0,0.1,0.2,0.25)$ single crystalline alloys, Phys. Chem. Chem. Phys., 2013, 15, 6965-6972.

32 A. H. Reshak, Ab initio study of TaON, an active photocatalyst under visible light irradiation, Phys. Chem. Chem. Phys., 2014, 16, 10558-10565.

33 I. M. Band, Y. I. Kharitonov and M. B. Trzhaskovskaya, Photoionization cross sections and photoelectron angular distributions for X-ray line energies in the range 0.1324.509 keV targets: $1 \leq Z \leq 100$, At. Data Nucl. Data Tables, 1979, 23, 443-505.

34 M. B. Trzhaskovskaya and V. G. Yarzhemsky, Dirac-Fock photoionization parameters for HAXPES applications, At. Data Nucl. Data Tables, 2018, 119, 99-174.
35 J. C. Slater and K. H. Johnson, Self-Consistent-Field X $\alpha$ Cluster Method for Polyatomic Molecules and Solids, Phys. Rev. B: Solid State, 1972, 5, 844-853.

36 M. V. Rama Rao and T. Shripathi, Photoelectron spectroscopic study of X-ray induced reduction of $\mathrm{CeO}_{2}$, J. Electron Spectrosc. Relat. Phenom., 1997, 87, 121-126.

37 E. Paparazzo, G. M. Ingo and N. Zacchetti, X-ray induced reduction effects at $\mathrm{CeO}_{2}$ surfaces: An X-ray photoelectron spectroscopy study, J. Vac. Sci. Technol., A, 1991, 9, 1416-1420.

38 F. Le Normand, J. El Fallah, L. Hilaire, P. Légaré, A. Kotani and J. C. Parlebas, Photoemission on 3d core levels of Cerium: An experimental and theoretical investigation of the reduction of cerium dioxide, Solid State Commun., 1989, 71, 885-889.

39 T. Hasegawa, S. M. Shahed, Y. Sainoo, A. Beniya, N. Isomura, Y. Watanabe and T. Komeda, Epitaxial growth of $\mathrm{CeO}_{2}(111)$ film on $\mathrm{Ru}(0001)$ : scanning tunneling microscopy (STM) and X-ray photoemission spectroscopy (XPS) study, J. Chem. Phys., 2014, 140, 044711.

40 Yu. A. Teterin and S. G. Gagarin, Inner valence molecular orbitals and the structure of X-ray photoelectron spectra, Russ. Chem. Rev., 1996, 65, 825-847.

41 R. S. Mulliken, Chemical Bonding, Annu. Rev. Phys. Chem., 1978, 29, 1-31. 